\title{
Endoscopic Submucosal Dissection for Gastric-phenotype Adenoma on the Surface of Gastric Gastrointestinal Stromal Tumor
}

\author{
Shumpei Yamamoto, Naoyuki Nishimura, Motowo Mizuno, Yuichi Shimodate, Akira Doi, \\ Hirokazu Mouri, Kazuhiro Matsueda and Hiroshi Yamamoto
}

\begin{abstract}
:
We herein report a rare case of a gastric adenoma overlying a gastrointestinal stromal tumor (GIST) that was removed by endoscopic submucosal dissection (ESD). A 78-year-old woman was referred to our hospital. Esophagogastroduodenoscopy revealed an elevated lesion of $15 \mathrm{~mm}$ in diameter overlying a submucosal mass in the gastric cardia. ESD was performed for the epithelial neoplasm, and biopsy specimens were obtained directly from the exposed surface of the submucosal tumor. The epithelial tumor was a tubular adenoma with focal severe atypia and a gastric phenotype. Biopsy specimens revealed a GIST, which was resected by laparoscopic intragastric surgery afterward.
\end{abstract}

Key words: endoscopic submucosal dissection, submucosal tumor, gastric-phenotype adenoma, gastrointestinal stromal tumor

(Intern Med 58: 201-205, 2019)

(DOI: 10.2169/internalmedicine.1122-18)

\section{Introduction}

The synchronous appearance of a gastric epithelial neoplasm and a submucosal tumor, including gastrointestinal stromal tumor (GIST) of the stomach, has seldom been reported (1-9). All reported cases were treated by one-time surgical gastric resection, and most underwent operation without a preoperative pathological diagnosis of the submucosal tumor (1-7). Endoscopic submucosal dissection (ESD) is accepted as a less-invasive method than gastrectomy for the treatment of early gastric cancer (10). ESD can achieve en-bloc margin-negative resection of superficial gastric neoplasms, regardless of their size and location (11).

We herein report a case of a gastric adenoma on the surface of a gastric GIST. We treated the adenoma by ESD and then resected the GIST by a laparoscopic intragastric operation.

\section{Case Report}

A 78-year-old woman was referred to our hospital for a neoplasm on the surface of a submucosal tumor in the posterior wall of the gastric cardia. During 10 years' surveillance, the submucosal tumor had gradually increased in size. At the most recent follow-up, however, an epithelial neoplasm was discovered, and she was referred to our hospital. Her serum was negative for Helicobacter pylori antibodies although she had no history of $H$. pylori eradication therapy.

Esophagogastroduodenoscopy (EGD) revealed a white, villous, elevated lesion of $15 \mathrm{~mm}$ in diameter overlying a round submucosal tumor of $20 \mathrm{~mm}$ in diameter in the posterior wall of the gastric cardia (Fig. 1a). Magnifying endoscopy with narrow-band imaging of the epithelial neoplasm revealed an irregular micro-surface pattern (Fig. 1b). A biopsy specimen of the lesion revealed a tubular adenoma with mild atypia. Endoscopic ultrasonography revealed the submucosal tumor to be a homogenous hypoechoic mass originating from the muscularis propria (Fig. 1c), and the 

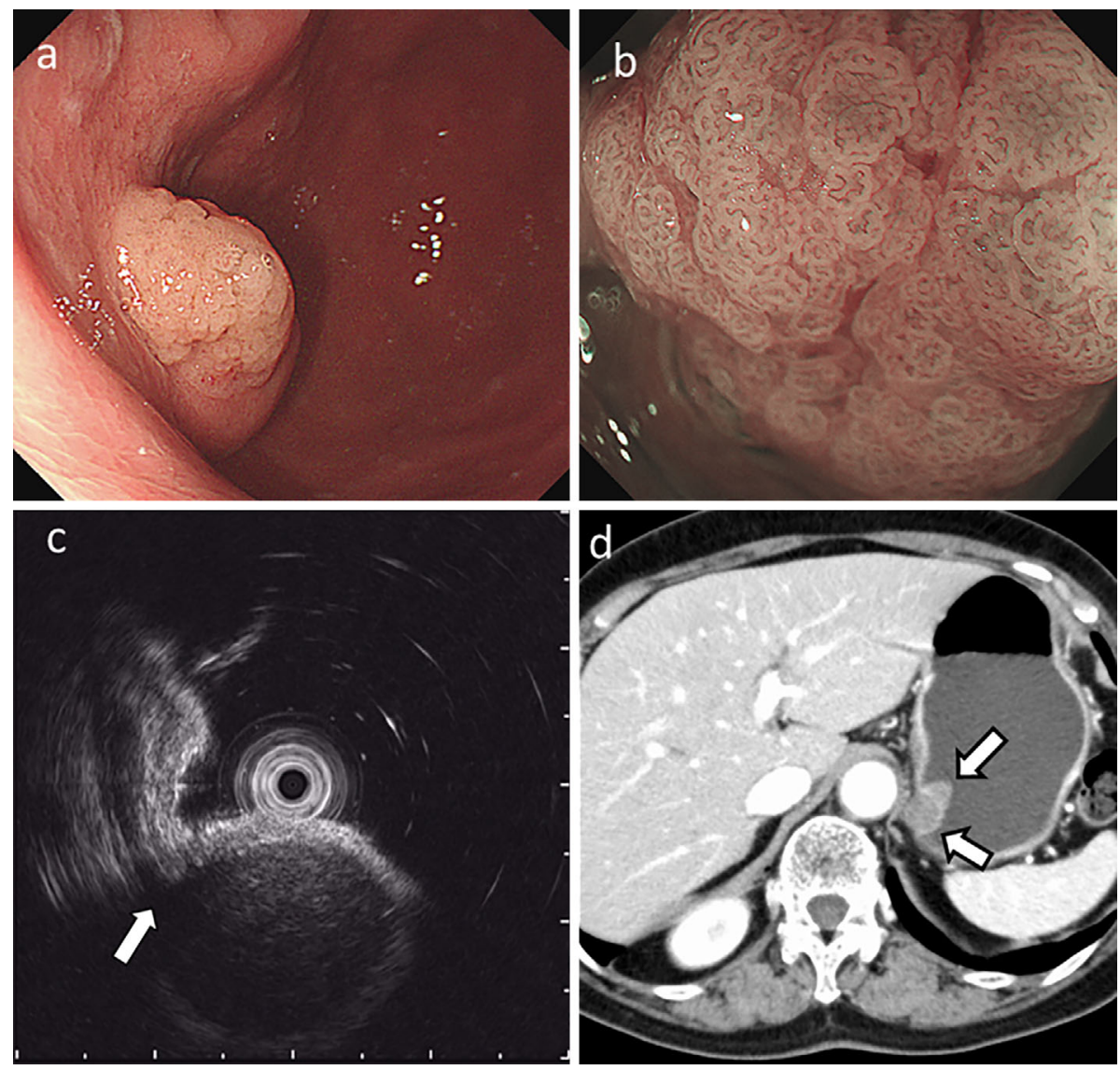

Figure 1. Images of endoscopy and computed tomography. a: White-light endoscopy shows a whitish villous elevated lesion on the side of submucosal tumor in the posterior wall of the gastric cardia. b: Magnifying endoscopy with narrow-band imaging of the epithelial neoplasm shows an irregular microsurface pattern. c: Endoscopic ultrasonography shows the submucosal tumor is a homogenous hypoechoic mass originating from the fourth layer of the gastric wall, i.e. the muscularis propria (arrows). d: Computed tomography shows an endoluminal low-density mass with a low-density nodule on its surface (arrows).

epithelial neoplasm was confined to the mucosal layer. Neither lesion had invaded the submucosal layer. The background gastric mucosa had inactive gastritis with moderate atrophy. Computed tomography revealed an endoluminal, homogenous, low-density mass with an overlying lowdensity nodule in the posterior wall of the gastric cardia (Fig. 1d). The radiologic differential diagnosis of the submucosal tumor was GIST, leiomyoma or schwannoma. An ultrasound-guided fine-needle aspiration biopsy of the submucosal tumor was not performed because of its technical difficulty due to the tumor location. We therefore planned resection of the gastric adenoma by ESD, a biopsy of the submucosal tumor through the resected base after ESD and therapy of the submucosal tumor according to its histopathological features.

ESD was performed with a dual Knife-J (Olympus, Tokyo, Japan), and direct biopsy specimens were obtained from the exposed surface of the submucosal tumor (Fig. 2a and b). The histological diagnosis of the resected adenoma $(15 \times 10 \mathrm{~mm})$ was tubular adenoma with focal severe atypia and negative horizontal and vertical margins. On an immunohistochemical examination, the tumor cells were strongly positive for MUC6, indicating a gastric phenotype (Fig. 3). The biopsy specimens of the underlying tumor revealed cells positive for C-KIT and CD34a and negative for $\alpha$-smooth muscle actin ( $\alpha$-SMA) and desmin; a diagnosis of GIST was thus made. According to the patient's wish, we performed follow-up EGD six months later and confirmed that the ulcer created by ESD had healed; the GIST was covered by regenerative mucosa (Fig. 2c). Using a two-port laparoscopic device placed in the right upper quadrant and the umbilicus, we resected the tumor. Hematoxylin and Eosin staining showed the resected tumor was localized within the submucosal layer (Fig. 4a). The tumor cells were strongly positive for C-KIT (Fig. 4b), and the Ki-67 labeling index was 3\% (Fig. 4c), indicative of a very low-risk GIST.

The patient's postoperative course was uneventful, and she had no recurrence of the neoplasm after six months' follow-up.

\section{Discussion}

To our knowledge, this is the first report of ESD performed for a gastric epithelial neoplasm on the surface of a 

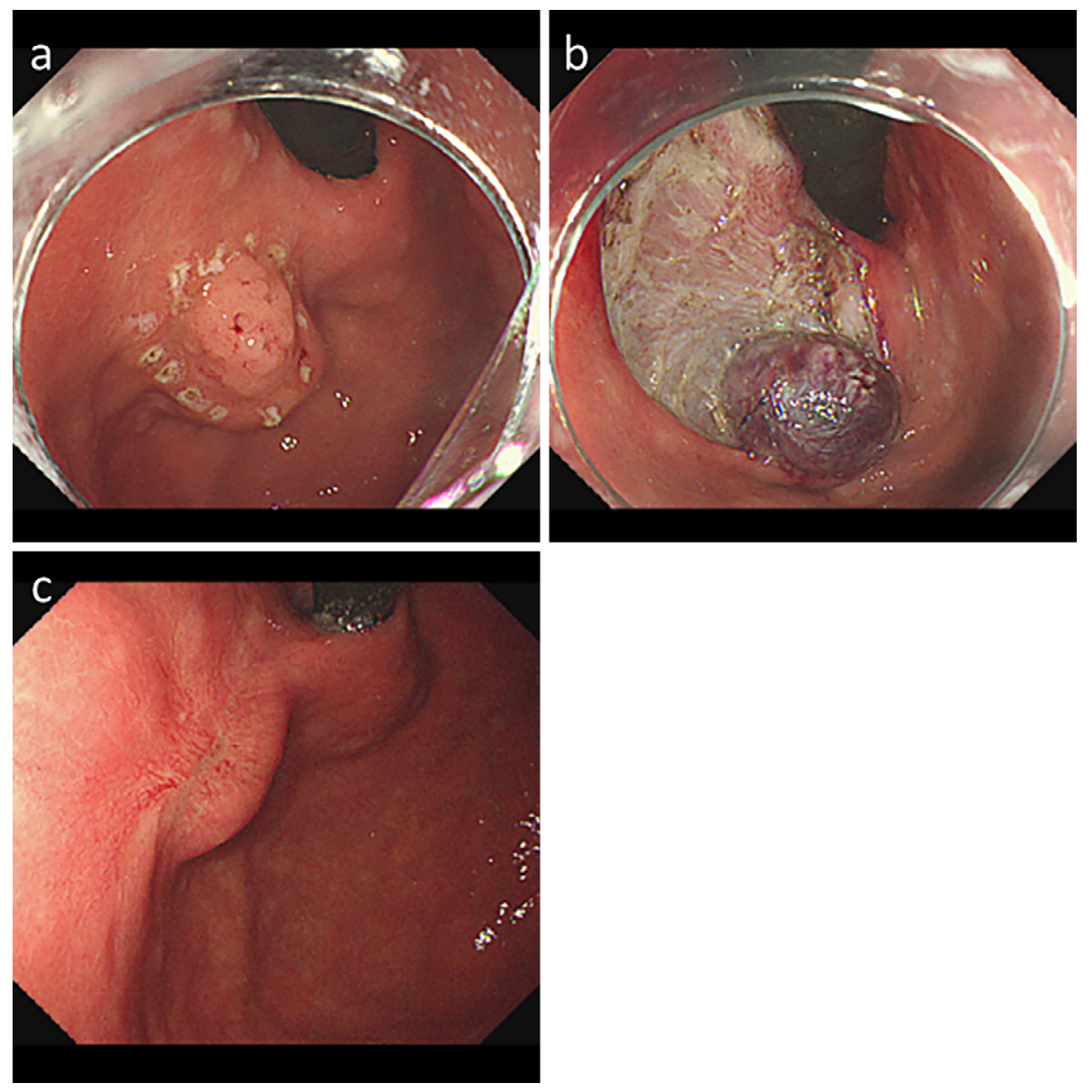

Figure 2. ESD of the gastric adenoma on the surface of the submucosal tumor. a: White markings are seen surrounding the intraepithelial neoplasm on the submucosal tumor. b: The exposed surface of the submucosal tumor after ESD is seen. c: The ulcer after ESD is healed and covered by the regenerative mucosa.


Figure 3. Histological findings of the resected epithelial neoplasm. a: Hematoxylin and Eosin staining of a whole-mount section shows tubular adenoma with focal severe atypia. b: Immunohistochemically, the tumor cells are strongly positive for MUC6. c: The tumor cells are negative for MUC2.

gastric GIST. This case has two interesting points: ESD was performed for a gastric epithelial neoplasm overlying a submucosal tumor, and a histological diagnosis was made by a biopsy through the exposed surface of the submucosal tumor after ESD.

Although there have been several reports of the synchronous appearance of gastric epithelial neoplasm and GIST (1-9), they were located in different locations of the stomach in most cases. Only three cases of gastric epithelial neoplasm and gastric GIST in the same location have been reported, and all were treated by gastrectomy (1-3). In our case, proximal gastrectomy was a therapeutic option. Although the biopsy of the epithelial neoplasm showed adenoma, the lesion was deemed likely to be a welldifferentiated adenocarcinoma based on its endoscopic characteristics, meaning that lymph node dissection would be necessary. However, given that the epithelial neoplasm was confined to the mucosal layer of the stomach, endoscopic resection was appropriate, and the patient wished for this less-invasive treatment. Another option was laparoscopy- 

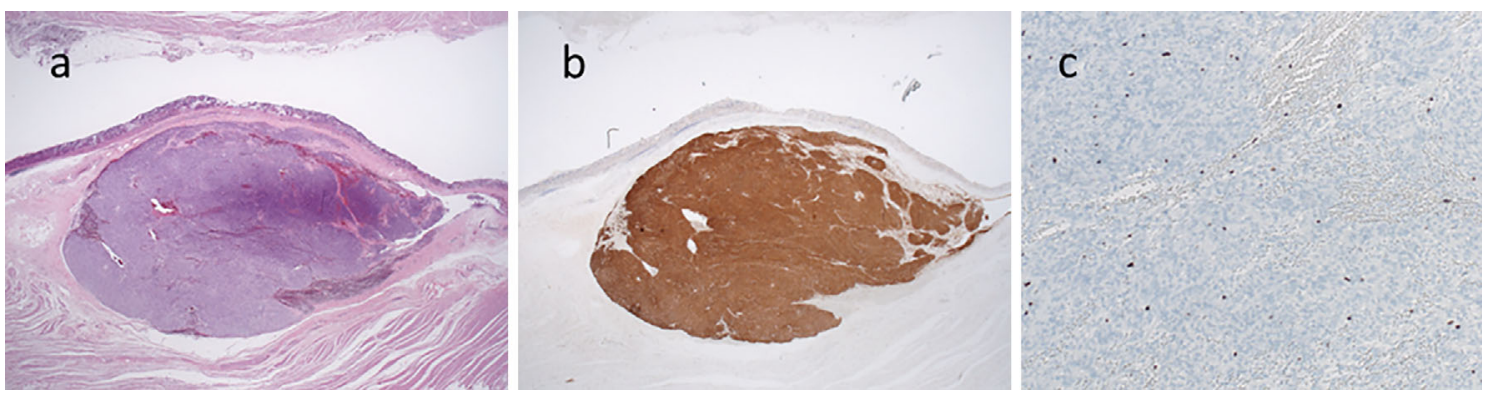

Figure 4. Histological findings of the resected GIST. a: Hematoxylin and Eosin staining of a whole mount section of the GIST. b: Immunohistochemically, the tumor cells are strongly positive for CKIT. c: The Ki-67 labeling index is $3 \%$.

endoscopy cooperative surgery, but this was not chosen due to the risk of peritoneal dissemination of the epithelial neoplasm during the procedure. Thus, we first performed ESD of the epithelial neoplasm. In addition, the submucosal tumor underneath had a possibility of being a GIST. To achieve complete resection of the epithelial neoplasm without damaging the submucosal tumor, we performed ESD carefully after confirming a lack of submucosal invasion of either the intraepithelial neoplasm or the submucosal tumor. En-bloc resection was achieved margin-negative without complication.

Another advantage of our treatment strategy was that the histological diagnosis of the GIST was made by an endoscopic biopsy of its exposed surface after ESD. We decided to resect the submucosal tumor which had gradually increased in size, based on a histological diagnosis of GIST. The usefulness and accuracy of a direct biopsy from a gastric submucosal tumor after exposing its surface by ESD has been reported (12).

Whether the synchronous appearance of gastric epithelial neoplasm and GIST is only a matter of simple coincidence or if the two lesions have a common causal relationship is unclear at present. $H$. pylori infection is a major cause of gastric cancer (13), but the association of its infection with GIST has not been elucidated. Yan et al. found no obvious relationship between gastric cancer and GIST according to the clinicopathological profiles and a molecular analysis of KIT/PDGFRA (6). However, Luo et al. showed that $c$-kit mutations were less common in GIST with synchronous adenocarcinoma than in GIST alone (7). Du et al. reported that patients with GIST and synchronous other gastrointestinal cancer including gastric cancer had a worse prognosis than those with GIST alone (5). These observations may imply an interaction between GIST and synchronous gastric cancer, but further studies are needed to better understand the mechanism underlying the synchronous development of these tumors of different histological types in the same organ.

Gastric epithelial neoplasm overlying a submucosal tumor is rare, but we believe that our experience in the present case will be useful to others treating such patients.
The authors state that they have no Conflict of Interest (COI).

\section{References}

1. Maiorana A, Fante R, Maria Cesinaro A, Adriana Fano R. Synchronous occurrence of epithelial and stromal tumors in the stomach: a report of 6 cases. Archi Pathol Lab Med 124: 682-686, 2000.

2. Katsoulis IE, Bossi M, Richman PI, Livingstone JI. Collision of adenocarcinoma and gastrointestinal stromal tumour (GIST) in the stomach: report of a case. Int Semin Surg Oncol 4: 2, 2007.

3. Telugu RB, Pushparaj M, Masih D, Pulimood A. Synchronous appearance of adenocarcinoma and gastrointestinal stromal tumour (GIST) of the stomach: a case report. J Clin Diagn Res 10: ED16ED18, 2016.

4. Narasimhamurthy MS, Vallachira GP, Mahadev PS. Synchronous adenocarcinoma and gastrointestinal stromal tumor in the stomach. Saudi J Gastroenterol 16: 218-220, 2010.

5. Du J, Shen N, He HS, Fu XL, Wang JZ, Mao CZ. Synchronous gastrointestinal cancer and gastrointestinal stromal tumors: a single-institution experience. World J Surg Oncol 14: 130, 2016.

6. Yan Y, Li Z, Liu Y, Zhang L, Li J, Ji J. Coexistence of gastrointestinal stromal tumors and gastric adenocarcinomas. Tumour Biol 34: 919-927, 2013.

7. Luo JM, Cao FL, Meng C, et al. Clinicopathological and molecular characteristics of synchronous gastric adenocarcinoma and gastrointestinal stromal tumors. Sci Rep 7: 12890, 2017.

8. Liu Y-J, Yang Z, Hao L-S, Xia L, Jia Q-B, Wu X-T. Synchronous incidental gastrointestinal stromal and epithelial malignant tumors. World J Gastroenterol 15: 2027, 2009.

9. Namikawa T, Munekage E, Munekage M, et al. Synchronous large gastrointestinal stromal tumor and adenocarcinoma in the stomach treated with imatinib mesylate followed by total gastrectomy. Anticancer research 36: 1855-1859, 2016.

10. Fukunaga S, Nagami Y, Shiba M, et al. Long-term prognosis of expanded-indication differentiated-type early gastric cancer treated with endoscopic submucosal dissection or surgery using propensity score analysis. Gastrointest Endosc 85: 143-152, 2017.

11. Yokoi C, Gotoda T, Hamanaka H, Oda I. Endoscopic submucosal dissection allows curative resection of locally recurrent early gastric cancer after prior endoscopic mucosal resection. Gastrointest Endosc 64: 212-218, 2006.

12. Kobara H, Mori H, Fujiwara S, Nishiyama N, Kobayashi M, Masaki T. Bloc biopsy by tunneling method using endoscopic submucosal dissection for an upper gastrointestinal submucosal tumor. Endoscopy 44 (Suppl): E197-E198, 2012.

13. Uemura N, Okamoto $\mathrm{S}$, Yamamoto $\mathrm{S}$, et al. Helicobacter pylori infection and the development of gastric cancer. N Engl J Med 345: 784-789, 2001. 
The Internal Medicine is an Open Access journal distributed under the Creative Commons Attribution-NonCommercial-NoDerivatives 4.0 International License. To view the details of this license, please visit (https://creativecommons.org/licenses/ by-nc-nd/4.0/).

(C) 2019 The Japanese Society of Internal Medicine

Intern Med 58: 201-205, 2019 